\title{
A review on rising bubble dynamics in viscosity-stratified fluids
}

\author{
KIRTI CHANDRA SAHU
}

Department of Chemical Engineering, Indian Institute of Technology Hyderabad, Sangareddy 502 285, India

e-mail: ksahu@iith.ac.in

MS received 25 May 2016; revised 2 September 2016; accepted 4 October 2016

\begin{abstract}
Systems with a bubble rising in a fluid, which has a variation of viscosity in space and time can be found in various natural phenomena and industrial applications, including food processing, oil extraction, waste processing and biochemical reactors, to name a few. A review of the aspects studied in the literature on this phenomenon, the gaps that exist and the direction for further numerical and experimental studies to address these gaps is presented.
\end{abstract}

Keywords. Multiphase flow; non-Newtonian; immiscible fluids; bubbles; numerical simulations.

\section{Introduction}

The fluid dynamics of a gas bubble rising due to buoyancy in a surrounding liquid (as shown in figure 1) has been studied from many centuries ago (the first reference in this subject goes back to Leonardo Da Vinci in the 1500 s). It continues to be a problem of interest even today due to its relevance in many industrial and natural phenomena (see e.g. [1-3]). They include aerosol transfer from sea, oxygen dissolution in lakes due to rain and electrification of atmosphere by sea bubbles, in bubble column reactors, in microfluidics, in the petroleum industry, for the flow of foams and suspensions and in carbon sequestration, to name a few. In many of these processes, viscosity of the fluid surrounding the bubble is not constant, but varies with space and time. This viscosity variation in turn greatly affects the rising dynamics of a gas bubble as compared with the ideal case of constant viscosity and density of the surrounding fluid. The latter case (ideal case) has been frequently studied in the past (see for instance [4]), and we hereafter term it as the "standard system". However, in real situations, due to the inherent presence of temperature variation and/or concentration gradient of species in the afore-mentioned processes, the viscosity of the surrounding fluid, as well as the surface tension at the interface separating the fluids, can vary drastically in space and time. For example, in the ice-cream and lotion industries, by the addition of only $2 \%$ carboxymethylcellulose (CMC) the viscosity of water or milk is made to increase by almost three orders of magnitude, while keeping the density constant [5]. The presence of temperature and concentration gradients also creates a variation of the surface tension normal and tangential to the interface separating the fluids, which leads to another class of complex flows in the vicinity of the interface, commonly known as Marangoni flows [6-8]. Yet another class of fluids are non-Newtonian fluids, where the viscosity depends on shear rate and/or yield stress, which in turn can be a function of space and time in a dynamically changing system [9].

The study of dynamics of an air bubble rising in liquids is difficult both numerically and experimentally, due to the high contrasts in fluid properties, dynamically changing interface between the fluids, presence of interfacial tension, etc. The time and length scales associated with this complex phenomenon, particularly at the time of topological change and break-up, are very small. This creates extra difficulties in numerical simulations and experiments. Thus, a great variety of numerical methods have been proposed, which range from boundary-fitted grids [10,11], to the level-set method [12, 13], the VOF method [14], diffuseinterface method [15], coupled level-set and volume-offluid method [16] and hybrid schemes of the latticeBoltzmann and the finite-difference method [17]. Also in the recent years, with the development of powerful highspeed cameras and sophisticated experimental facilities, several researchers continue to perform experiments in this area (see e.g. [18, 19]). The first systematic experiment of a rising air bubble in liquids was conducted by Bhaga and Weber [20]. As this review is limited to summarizing the physical phenomena associated with the rising of an air bubble in viscosity-stratified fluids, the reader is referred to the afore-mentioned papers for the details on various numerical methods and experimental techniques used to study this complex problem.

In this review, the fluid dynamics of a gaseous bubble inside viscosity-stratified fluids is discussed. In order to keep the system simple and isolate the influence of viscosity stratification only, the densities of the fluids are assumed to be constant and the flow is assumed to be incompressible. Also this review is limited to the studies of 


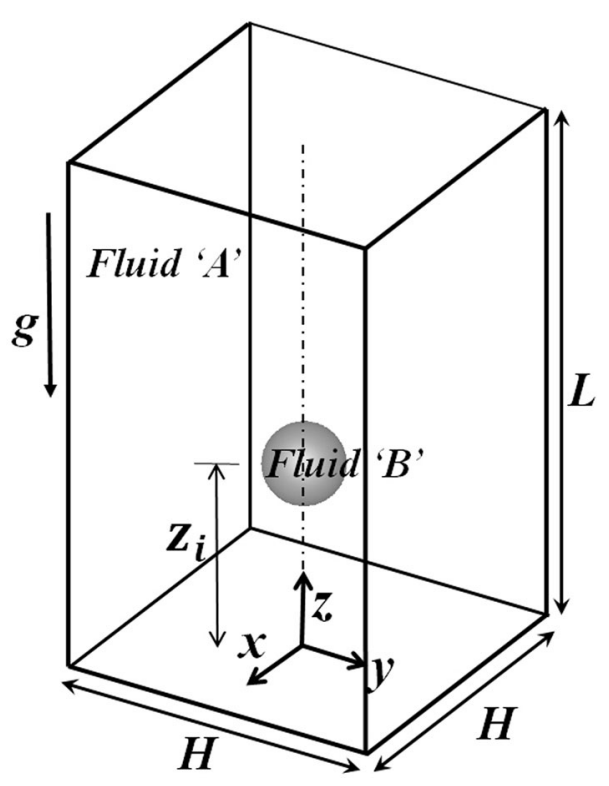

Figure 1. Schematic diagram of a bubble (fluid 'B') rising inside a viscosity stratified medium (fluid ' $A$ ') under the action of buoyancy. The gravity is acting in the negative $z$-direction.

single bubble and two bubbles only. There are also several studies in the literature that considered the dynamics of many bubbles simultaneously (e.g. studies associated with bubble-column reactors). The studies involving many bubbles are not discussed in the present review.

\subsection{Brief discussion on "standard" systems (constant viscosity of the surrounding fluid)}

Before discussing what happens in viscosity-stratified systems, it is useful to understand the bubble rise phenomena in the "standard" systems. Here, we will also define some dimensionless parameters, which will be used throughout this review. In the dimensionless formulation, the rising bubble problem is completely described by four non-dimensional parameters, namely, the Galilei number $G a\left(\equiv \rho_{B} V R / \mu_{B}\right)$, the Eötvös number $E o\left(\equiv \rho_{B} g R^{2} / \sigma\right)$, the density ratio $\rho_{r} \equiv \rho_{A} / \rho_{B}$ and the viscosity ratio $\mu_{r} \equiv \mu_{A} / \mu_{B}$, wherein $\sigma$ is surface tension, $\mu_{A}, \rho_{A}$ and $\mu_{B}, \rho_{B}$ are the viscosity and density of fluids ' $\mathrm{A}$ ' and ' $\mathrm{B}$ ', respectively (as shown as figure 1). $V=\sqrt{g R}$, wherein $g$ is the acceleration due to gravity and $R$ is the equivalent radius of the bubble. Another dimensionless number, the Morton number, $M o \equiv E o^{3} / G a^{4}$, which depends on fluid properties only, but not on the radius of the bubble, will also be used sometime in the following sections.

A number of numerical and experimental studies have been conducted on single bubble rising in quiescent liquid in the "standard" systems (see for instance [20, 23-29]). Most of these earlier studies investigated terminal velocity,

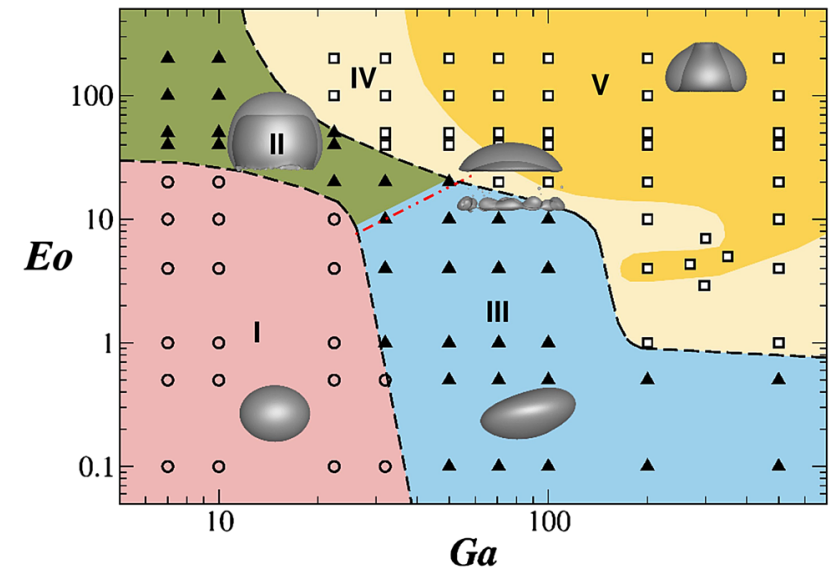

Figure 2. Behaviour of a single bubble rising in quiescent liquid in a "standard" system. The red dash-dotted line is the $M o=10^{-3}$ line, which separates the regions of skirted bubble (region II) and oscillatory bubble (region III). This figure is taken from Tripathi et al [4].

deformation and path instability associated with the rising bubble inside another fluid. Recently, Tripathi et al [4] revisited this problem by conducting three-dimensional numerical simulations, and identified regimes of starkly distinct behaviours in the Galilei $(G a)$ and Eötvös (Eo) numbers plane (as shown in figure 2). They found that for low $G a$ and $E o$ (region I) the bubble maintains azimuthal symmetry. In region II (low $G a$ and high Eo), the bubble forms a skirt, and in region III (for high $G a$ and low Eo), the bubble moves in a spiral or a zigzag path. Vortex shedding is observed in the wake of region III bubbles. For high values of $G a$ and $E o$, the bubble breaks to form satellite bubbles (region IV) or undergoes topological change to form toroidal shape (region V). However, it is to be noted here that the phase diagram presented by Tripathi et al [4] (shown in figure 2) corresponds to an initially spherical gaseous bubble in water, and a different initial condition may alter the boundaries separating these regions. It is an important point because creating a spherical shape bubble initially is a difficult task in experiments, particularly for large bubbles. In another study, Tsamopoulos et al [22] provided a library of terminal shapes of a bubble rising in a liquid in the $G a-E o$ plane for a "standard" system (see figure 3) by performing time-independent axisymmetric numerical simulations.

Another important aspect is the influence of shape change on the drag and lift forces acting on a rising bubble. Hadamard [30] reported that the drag forces observed in spherical bubbles are different from that of solid spheres due to the internal flow in bubbles. Later, Taylor and Acrivos [23] analytically derived the first-order correction to the drag force of a slightly deformed spheroidal bubble in the creeping flow limit. For bubbles rising non-axisymmetrically (region III bubbles), a sideways motion can ensue. The force responsible for this asymmetric motion is 


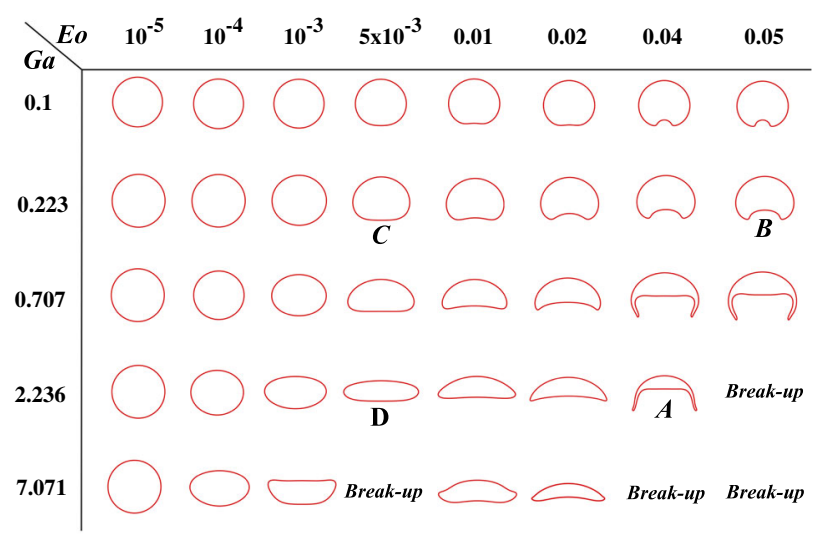

Figure 3. The terminal shapes of the bubble obtained from the present simulations in $G a-E o$ space for the "standard" system. The rest of the parameter values are $\rho_{r}=10^{3}$ and $\mu_{0}=10^{2}$. This figure is taken from Premlata et al [21]. A similar figure was also given by Tsamopoulos et al [22], who conducted steady-state simulations.

the lift force. Both the lift and the drag forces are highly dependent on the shape of the rising bubble. Several researchers [31, 32] have studied the variations of these forces as a function of aspect ratio of the bubble.

\section{Governing equations}

An initially spherical air bubble (designated by fluid 'B') of initial radius $R$, having constant viscosity $\mu_{B}$ rising under the action of buoyancy in another fluid (designated by fluid 'A') is considered, as shown in figure 1. Both the fluids are considered to be incompressible. In the Cartesian coordinates system $(x, y, z)$, the dimensionless governing equations of mass and momentum conservation are given by

$$
\begin{gathered}
\nabla \cdot \mathbf{u}=0 \\
\rho\left[\frac{\partial \mathbf{u}}{\partial t}+\mathbf{u} \cdot \nabla \mathbf{u}\right]=-\nabla p+\frac{1}{G a} \nabla \cdot\left[\mu\left(\nabla \mathbf{u}+\nabla \mathbf{u}^{T}\right)\right] \\
+\frac{\delta}{E o} \hat{n} \nabla \cdot \hat{n}-\rho \mathbf{e}_{z} .
\end{gathered}
$$

In the framework of volume-of-fluid ( $\mathrm{VoF})$ method, the equation for the volume fraction, $c$, of the fluid ' $\mathrm{A}$ ', whose values are 0 and 1 for the air and liquid phases, respectively, is given by

$$
\frac{\partial c}{\partial t}+\mathbf{u} \cdot \nabla c=0
$$

Here $\mathbf{u}(u, v, w)$ represents velocity field, wherein $u, v$ and $w$ are the velocity components in the $x, y$ and $z$ directions, respectively; $p$ denotes the pressure field; $\delta$ is the Dirac delta function; $\kappa=\nabla \cdot \mathbf{n}$ is the curvature, $\mathbf{n}$ is the unit normal to the interface pointing towards fluid ' $A$ ', $\mathbf{e}_{z}$ represents the unit vector in the vertically upward direction and $\sigma$ is the interfacial tension coefficient of the liquid-gas interface. The above equations are non-dimensionalized by using the initial radius of bubble, $R$, and $\sqrt{g R}$ as the length and velocity scales, respectively. The dimensionless density $\rho$ is given by

$$
\rho=(1-c) \rho_{r}+c,
$$

and the viscosity field is given by

$$
\mu=(1-c) \mu_{r}+c .
$$

Although governing equations associated with a VoF method are given above, there are several other numerical approaches, such as the level-set method [12, 13], diffuseinterface method [15], coupled level-set and volume-offluid method [16], hybrid schemes of the lattice-Boltzmann and the finite difference method [17], etc., which are also frequently used to study such interfacial problems. Each of the afore-mentioned method has certain advantages and disadvantages, and is selected based on parameter range considered and/or the problem at hand.

\section{Discussion}

As discussed in the introduction, the objective of this work is to present the dynamics of a bubble in viscosity-stratified media. Three situations are considered, namely (i) variation of viscosity of the surrounding fluid in space due to some species, (ii) viscosity stratification of the surrounding fluid due to the presence of temperature gradient and (iii) viscosity variations due the non-Newtonian nature of the fluid surrounding the bubble. They are discussed one-by-one in the following subsections.

\subsection{Viscosity variation of the surrounding fluid in space}

Temperature and concentration variations lead to continuous viscosity-stratifications. In such systems, the rising bubble dynamics is not only affected by viscosity stratification, but also greatly influenced by the variations of surface tension across and along the interface separating the fluids. There are several situations where the viscosity stratification is inherent, i.e., not due to the presence of temperature and concentration gradients. In many applications [9], immiscible fluid layers of almost the same density but significant viscosity jump are observed. A gaseous bubble rising through an interface separating two immiscible liquids of different viscosities has been studied in the past by several researchers [33-35]. As the bubble crosses the interface, the bottom fluid migrates along the wake of the bubble to the upper fluid, having a different viscosity, which in turn changes the dynamics of the bubble as compared with the "standard" system. 
40

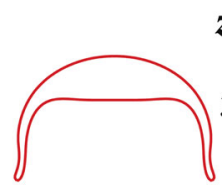

30

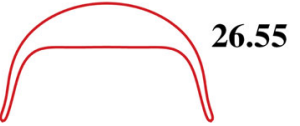

20

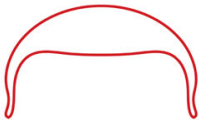

17.50

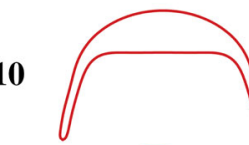

8.46

$t=\mathbf{0}$

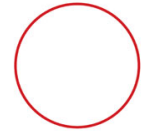

(a)

0.01
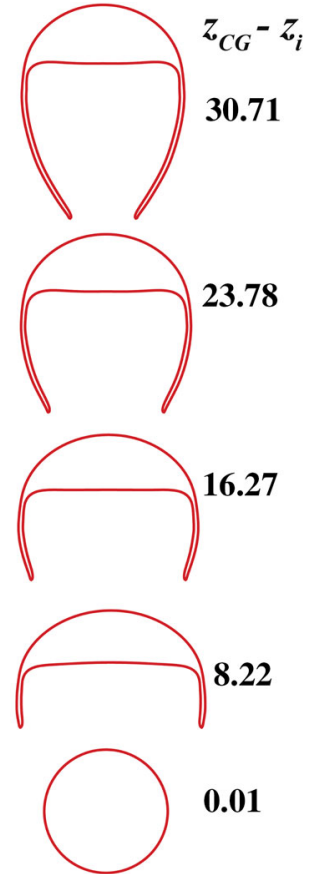

(b)

Figure 4. Time evolution of bubble shapes for $G a=2.236, E o=0.04$ : (a) constant viscosity system $\left(a_{1}=\right.$ $\left.1, a_{2}=0\right)$ and (b) linearly increasing viscosity $\left(a_{1}=0.2, a_{2}=0.2\right)$. This figure is taken from Premlata et al [21].

We discuss the rising bubble dynamics in a simpler system, wherein the viscosity of the surrounding fluid continuously increases in the vertical direction in the absence of Marangoni stresses, and other complicated physics discussed earlier. Consider a system where the viscosity of the surrounding fluid increases linearly in the vertical direction, $z$, such that

$$
\mu_{A}=\mu_{0}\left(a_{1}+a_{2} z\right),
$$

where $a_{1}$ and $a_{2}$ are constants and $\mu_{0}$ is the viscosity of surrounding fluid at a reference location, $z=z_{i}$. In this configuration, as the viscosity of the surrounding fluid is varying, the viscosity ratio can be defined as $\mu_{r 0} \equiv \mu_{0} / \mu_{B}$. Now, let us contrast the dynamics of a bubble in this viscosity stratified medium with that in the "standard" system. The parameter values considered for this comparison are $\mu_{r 0}=10^{-2}, \rho_{r}=10^{-3}, G a=2.236$ and Eo $=0.04$. This set of parameter values corresponds to point $\mathrm{A}$ in figure 3, which is taken from Tsamopoulos et al [22].

In figure 4(a) and (b), the temporal evolution of bubble shapes is presented for $G a=2.236$ and $E o=0.04$ for the standard system and a medium with linearly increasing viscosity, respectively. When we compare the shapes of the bubble at different dimensionless times, it can be seen that for the case of the linearly increasing viscosity medium (figure 4(b)) an elongated skirt (longer than what appears for the constant viscosity case (figure $4 a$ ) is formed. This is

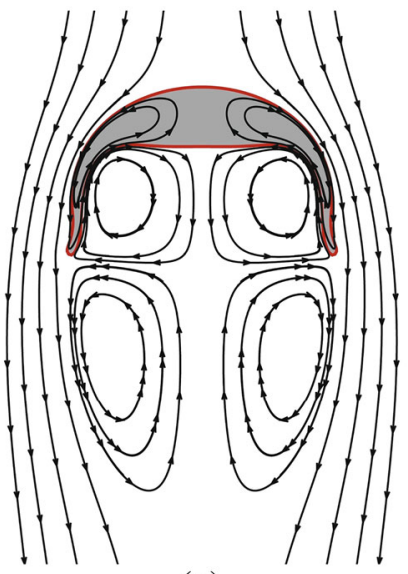

(a)

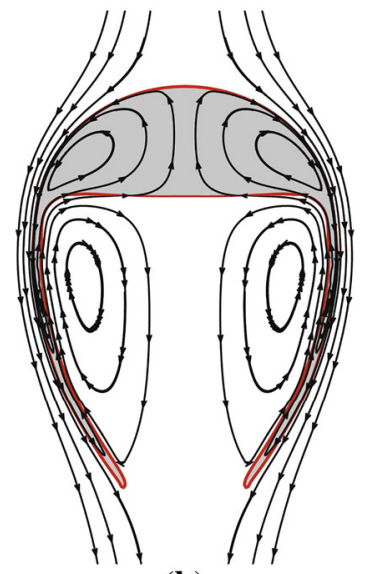

(b)
Figure 5. Streamlines at $t=40$ for (a) constant viscosity system and (b) linearly increasing viscosity. The remaining parameter values are the same as those used to generate figure 4 . This figure is taken from Premlata et al [21].

unexpected due to the following reason. As the bubble migrates in the vertical direction the local $G a$ decreases due to an increase in the local viscosity of the surrounding fluid. In figure 3, we can see that for $E o=0.04$, with a decrease in $G a$ the bubble changes its shape from a skirted (at $G a=2.236$ ) to a dimpled ellipsoidal (at $G a=0.1$ ). By lowering the value of $G a$ further we will get a spherical shape bubble (not shown). This observation is for the "standard" system. The dynamics is different in the viscosity-stratified system, wherein as the bubble rises in the upward direction, the less viscous fluid from the bottom part of the domain advects along with the recirculation region (inside the skirted region). This leads to a continuous increase in the viscosity contrast between the inside and outside regions separated by the skirt. The resultant stresses due to this differential viscosity on both sides of the skirt force it to curl inwards. This separation of fluids due to the skirt allows fast recirculation to occur in the fluid captured inside the wake of the bubble, while simultaneously allowing a slow flow outside the skirt. This creates a contrast in the inertia across the skirt. The streamlines at $t=40$ are plotted in figure 5(a) and (b) for the systems with constant viscosity and linearly increasing viscosity, respectively. It can be seen that for the constant viscosity system there are two recirculation zones in the wake region of the bubble, whereas only one recirculation zone appears in the wake region (enclosed by the skirt) of the bubble in linearly increasing viscosity medium. The appearance of two recirculation zones in constant viscosity case prevents the closing of the skirt, which can be seen in the linearly increasing viscosity medium. It is also to be noted here that the drag force is more in case of the bubble having a longer skirt (in case of linearly increasing fluid) as compared with that observed in the constant viscosity medium. This phenomenon may be beneficial to certain processes where a 
less viscosity fluid is required to be carried through a highly viscous fluid with the help of a carrier bubble. More discussion on this subject can be found in Premlata et al [21].

\subsection{Viscosity variation due to temperature gradient}

The pioneering work of Young et al [6] was the first to investigate the migration of bubbles due to thermal gradient. They investigated a system where the temperature of the surrounding viscous fluid decreases in the vertically upward direction, and found that small bubbles move in the downward direction, even though buoyancy acts in the upward direction. In this situation, the thermocapillary force and buoyancy force act in the opposite direction. In case of a small bubble the latter force dominates the former one, whereas for bigger bubbles, buoyancy wins, which causes the bubble to migrate in the upward direction. They also provided a theoretical description assuming spherical bubble and creeping flow conditions, which predicted the terminal velocity obtained in their experiment. Since then several theoretical analyses were performed to study thermocapillary migration of a bubble in the limit of both small and large Reynolds numbers, but neglecting buoyancy [36-40]. Later, Merritt et al [41] numerically investigated the dynamics of a bubble under the combined action of buoyancy and thermocapillarity forces. Zhang et al [42] also performed a theoretical analysis of a rising bubble for small Marangoni numbers under the influence of gravity.

The surface tension of common fluids, such as air, water and various oils, decreases almost linearly with increase in temperature. Here, these fluids are referred to as "linear" fluids. All the above-mentioned investigations considered the bubble dynamics in common/linear fluids. However, there is another class of fluid that exhibit a non-monotonic dependence of the surface tension on temperature. These fluids are known as "self-rewetting" fluids, which are nonazeotropic high carbon alcohol solutions [43-47]. These fluids were first studied by Vochten and Petre [43] who observed the occurrence of a minimum in surface tension with temperature in high carbon alcohol solutions. Later, Abe et al [48] named them as "self-rewetting" fluids. Recently, Tripathi et al [8] studied the buoyancy-driven rise of a bubble inside a tube imposing a constant temperature gradient along the wall and containing a liquid, whose surface tension has a quadratic dependence on temperature given as

$$
\sigma=\sigma_{0}\left[1-M_{1} T+M_{2} T^{2}\right]
$$

where $\sigma_{0}$ is the reference value of surface tension at the reference temperature, $T_{0} . \quad M_{1}\left(-\left.\frac{1}{\sigma_{0}} \frac{d \sigma}{d T}\right|_{T_{0}}\right) \quad$ and $M_{2}\left(\left.\frac{1}{\sigma_{0}} \frac{d^{2} \sigma}{d T^{2}}\right|_{T_{0}}\right)$ are Marangoni numbers associated with the linear and quadratic terms, respectively. For isothermal

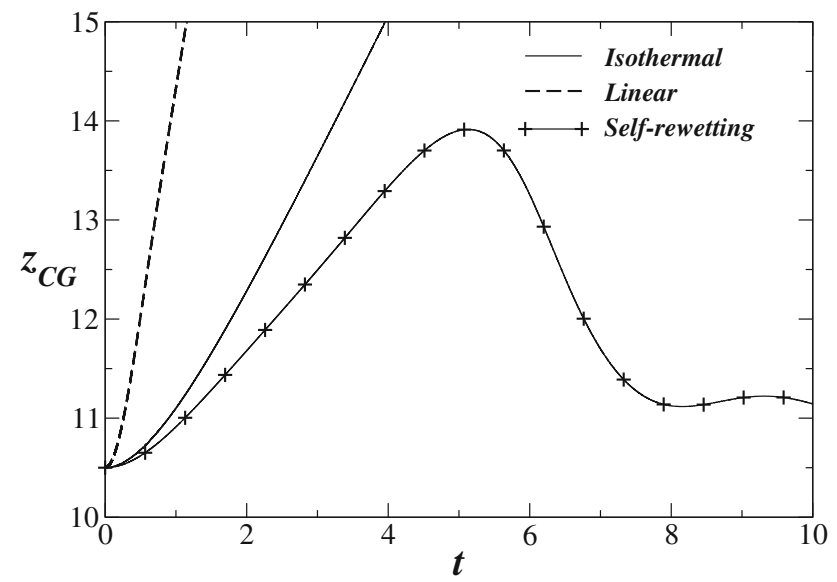

Figure 6. Migration of bubble in a isothermal system, linear (common) fluid and self-rewetting fluid for $G a=10, E o=10^{-2}$, $\rho_{r}=10^{-3}, \mu_{r}=10^{-2}$, the temperature gradient, $\Gamma=0.1$ and thermal diffusivity ratio $\alpha_{r}=0.04$. For isothermal system $M_{1}=0$ and $M_{2}=0$, for linear, $M_{1}=0.4$ and $M_{2}=0$ and for selfrewetting fluid, $M_{1}=0.4$ and $M_{2}=0.2$. This figure is taken from Tripathi et al [8].

systems, $M_{1}=M_{2}=0$, for common or linear fluids: $M_{1}$ is non-zero, but $M_{2}$ is zero, and for self-rewetting fluids, both $M_{1}$ and $M_{2}$ are non-zero.

The temporal variations of the centre of gravity, $z_{C G}$, of a rising bubble for three different cases: the isothermal case, and the cases of a simple linear fluid, and a self-rewetting fluid are shown in figure 6 . The temperature of the tube is maintained with a linear temperature profile of constant gradient $\Gamma>0$. It can be seen that the terminal velocity is higher for the non-isothermal case as compared with the isothermal system due to the presence of Marangoni stresses driving liquid towards the cold region of the tube and thereby enhancing the upward motion of the bubble. For the self-rewetting fluid in the non-isothermal case, the bubble reaches a constant speed for a certain time duration (rising phase), which is followed by downward motion (motion reversal). The bubble gets arrested eventually. This dynamics might be of interest to researchers working in microfluidics and multiphase microreactors.

\subsection{Viscosity variation due to the non-Newtonian nature of the surrounding fluid}

Another instance in which one could experience viscosity stratification is when the surrounding fluid is a non-Newtonian fluid. In this situation, viscosity can depend on the shear and its history [49]. The dynamics of a rising bubble in surrounding fluids that exhibit a non-Newtonian nature is important in many engineering applications, including food processing, oil extraction, waste processing and biochemical reactors. A range of non-Newtonian fluids can be considered, such as power-law and Bingham plastic fluids, 
whose behaviour is more complex than those of relatively simple fluids (such as water, silicone oil, etc.). However, this review is restricted to only bubble rise inside viscoplastic and shear-thinning fluids.

3.3a Viscoplastic fluid: Yield stress fluids or viscoplastic materials behave like both fluid and solid. If the stress is beyond a critical value, known as yield stress, these materials flow like liquids when subjected to stress, but behave as a solid below this critical level of stress. An extensive review on yield stress fluids can be found in $[50,51]$. Bingham [52] was the first to propose a constitutive law to describe yield stress fluids. Few years later, Herschel and Bulkley [53] extended this law to include the shear-thinning/thickening behaviour. According to this model, the material can be yielded (unyielded) if the stress is higher (lower) than the yield stress of the material. It is to be noted here that at the boundary separating the yielded and unyielded regions, the model becomes singular, where the viscosity of the fluid has an extremely sharp jump. Frigaard and Nouar [54] proposed, a simpler way to overcome this singularity by introducing a 'regularization' parameter. The regularized models were used several researchers to model the viscosity of non-Newtonian fluids $[22,55,56]$.

The motion of air bubbles in viscoplastic materials has been studied by many research groups in the past. Astarita and Apuzzo [57] was the first to conduct an experimental study on rising bubbles in viscoplastic materials (by considering Carbopol solutions). By varying the concentration of Carbopol in the solution (thereby varying the yield stress of the material), they investigated the shapes and rise velocities of the bubble. Since then, several researchers (e.g. $[19,58])$ have experimentally investigated the rising bubble phenomenon in viscoplastic materials.

Several researchers also studied this problem by conducting theoretical analysis (see e.g. [59]). Recently, Tsamopoulos et al [22] performed a detailed time-independent numerical study of a bubble rise phenomena, using the regularized Papanastasiou [60] model. They presented a library of bubble and yield surface shapes for a wide range of dimensionless parameters, taking into account the effects of inertia, surface tension and gravity. The shapes and unyielded surfaces of a bubble rising in a Bingham fluid as a function of Bond $\left(\equiv \rho_{A} g R^{2} / \sigma\right)$ and Archimedes ( $\equiv$ $\left.\rho_{A}^{2} g R^{3} / \mu_{A}^{2}\right)$ numbers are given in figure 7 . They found that when the yield stress of the surrounding Bingham plastic fluid is large the bubble does not move at all. This work was followed by the study of Dimakopoulos et al [61], who used the augmented Lagrangian method to obtain a more accurate estimation of the stopping conditions than that of Tsamopoulos et al [22]. It is to be noted here that Tsamopoulos et al [22] were not able to calculate steady shapes for a certain set of parameters, which is probably an indication that the flow may have become time-dependent in these conditions. Potapov et al. [62] and Singh and Denn

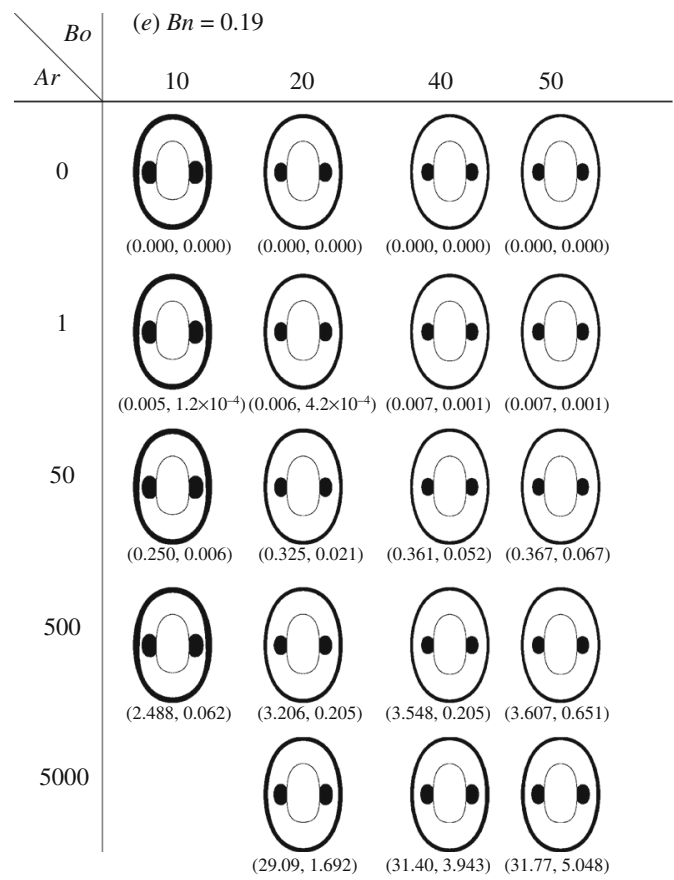

Figure 7. The steady-state shapes and unyielded surfaces of a bubble in Bond number and Archimedes number space for Bingham number $B n\left(\equiv \tau / \rho_{A} g R\right)=0.19 ; \tau$ is the yield stress of the material. The black surfaces represents the unyielded regions. The numbers written below each figures are Reynolds and Weber numbers, respectively. This figure is taken from Tsamopoulos et al [22].

[63] investigated the bubble dynamics in viscoplastic fluids through time-dependent simulations in the creeping flow conditions. Recently, Tripathi et al [56] performed unsteady simulations in the inertial regime of the buoyancy-driven rise of an air bubble inside an infinitely extended viscoplastic medium by considering the regularized Herschel-Bulkley model for the surrounding fluid, which is given by

$$
\mu=\frac{\tau_{0}}{\Pi+\epsilon}+\mu_{0}(\Pi+\epsilon)^{n-1},
$$

where $\tau_{0}$ and $n$ are the yield stress and flow index, respectively, $\epsilon$ is a small regularization parameter, and $\mu_{0}$ is the fluid consistency; $\Pi=\left(E_{i j} E_{i j}\right)^{1 / 2}$ is the second invariant of the strain rate tensor, wherein $E_{i j} \equiv \frac{1}{2}\left(\partial u_{i} / \partial x_{j}+\partial u_{j} / \partial x_{i}\right)$. They found that in the presence of inertia and in the case of weak surface tension the bubble does not reach a steady state and the dynamics may become complex for sufficiently high yield stress of the material.

3.3b Shear-thinning fluid: The peculiar physics associated with rising bubble in shear-thinning fluid due to the viscosity variation near the bubble surface has been a subject of research of several researchers [64, 65]. An extensive review on this subject is given by Chhabra $[66,67]$. Various 


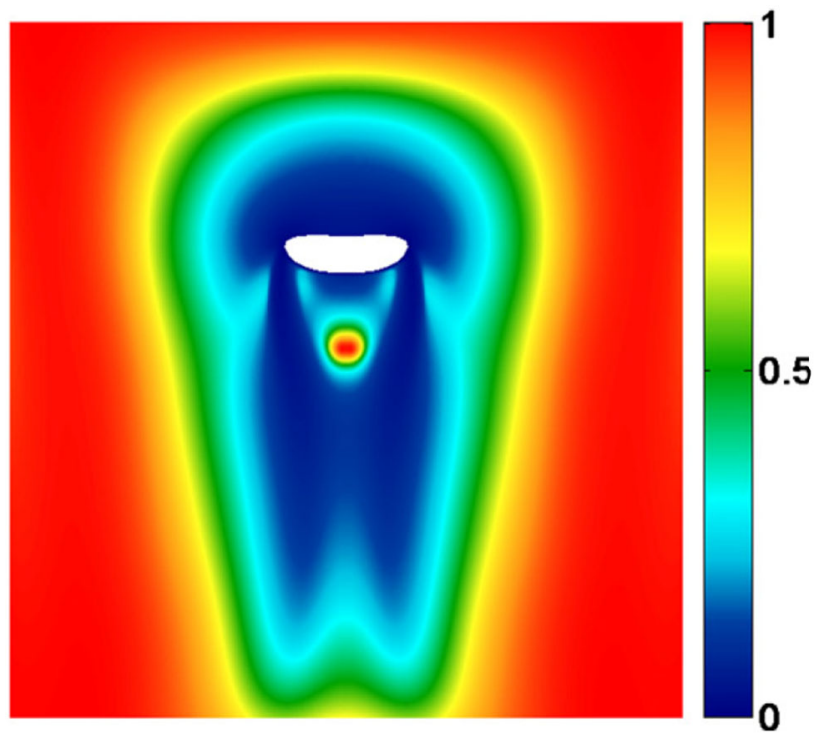

Figure 8. A typical distribution of viscosity and shape of the bubble in Carreau shear-thinning fluid. This figure is taken from Zhang et al [18].

numerical methods, such as volume-of-fluid (VoF) [68, 69], level set [70] and lattice Boltzmann [71], have been developed to improve the accuracy of the numerical procedure to investigate these problems. Recently, Zhang et al [18] experimentally and numerically investigated the dynamics of a rising bubble in a quiescent shear-thinning fluid, such as solutions of CMC, sodium hydroxyl-ethyl cellulose (HEC) and xanthan gum. They used the Carreau rheological model to describe the viscosity of the surrounding fluids. They found that the flow patterns around the bubble in shear-thinning fluids are very different from the one observed when the surrounding liquid is a Newtonian fluid. They also found the viscosity in the wake region is large as compared with the other part of the surrounding fluid, which leads to these differences in the flow pattern and deformation of the bubble as compared with those in the Newtonian case. The viscosity variation along with the shape of the bubble showing the afore mentioned features is presented in figure 8 . There are also a few studies (see e.g. $[72,73])$ that investigated the dynamics of a pair of bubbles aligned in different ways inside non-Newtonian fluids. They found that the irregularity of the bubble shape (deviation from the spherical shape) increases with an increase in the shear-thinning tendency. This effect was found to be significant for flow index $n<0.5$.

\section{Concluding remarks}

In this review, the dynamics of bubble rise in viscositystratified media is discussed. Many peculiar phenomena observed in such systems are presented, which are found to be very different from those observed when the surrounding fluid has a constant viscosity. Three configurations, wherein viscosity stratifications are due to the presence of a species, temperature gradient and non-Newtonian surrounding fluids, are considered. Although, dynamics of rising bubble in constant viscosity fluids has a long history, the dynamics in viscosity stratified media has not been investigated to that detail due to the requirement of powerful computers and sophisticated experimental techniques. The numerical simulations of such complex phenomena involving high density and viscosity ratios along with other physics are extremely difficult and computationally very expensive. Due to the improvement of advanced scientific computing resources and the development of sophisticated experimental techniques, it is possible to study such complex phenomena to greater depth at present.

\section{Acknowledgements}

The author gratefully acknowledges Prof Karri Badarinath of IIT Hyderabad for reading the manuscript and providing valuable suggestions. He also thank his $\mathrm{PhD}$ student A R Premlata for her help in re-plotting some of the results presented in this review.

\section{References}

[1] Blanchard D C 1962 Comments on the breakup of raindrops. J. Atmos. Sci. 19: 119-120

[2] Gal-Or B, Klinzing G E and Tavlarides L L 1969 Bubble and drop phenomena. Ind. Eng. Chem. 61(2): 21-34

[3] Villermaux E and Bossa B 2009 Single-drop fragmentation determines size distribution of raindrops. Nat. Phys. 5: 697-702

[4] Tripathi M K, Sahu K C and Govindarajan R 2015 Dynamics of an initially spherical bubble rising in quiescent liquid. Nat. Commun. 6: 6268

[5] Ghannam M T and Esmail M N 1997 Rheological properties of carboxymethyl cellulose. J. Appl. Polym. Sci. 64(2): 289-301

[6] Young N O, Goldstein J S and Block M J 1959 The motion of bubbles in a vertical temperature gradient. J. Fluid Mech. 6: $350-356$

[7] Subramanian R S, Balasubramaniam R and Wozniak G 2002 Fluid mechanics of bubbles and drops. In: Monti R (Ed.), Physics of fluids in microgravity. London: Taylor and Francis, pp. 149-177

[8] Tripathi M K, Sahu K C, Karapetsas G, Sefiane K and Matar O K 2015 Non-isothermal bubble rise: non-monotonic dependence of surface tension on temperature. J. Fluid Mech. 763: 82-108

[9] Govindarajan R and Sahu K C 2014 Instabilities in viscositystratified flows. Ann. Rev. Fluid Mech. 46: 331-353.

[10] Chen J C and Lee Y T 1992 Effect of surface deformation on thermocapillary bubble migration. AIAA J. 30(4): 993-998.

[11] Welch S W 1998 Transient thermocapillary migration of deformable bubbles. J. Colloid Interface Sci. 208(2): 500-508 
[12] Haj-Hariri H, Shi Q and Borhan A 1997 Thermocapillary motion of deformable drops at finite Reynolds and Marangoni numbers. Phys. Fluids 9(4): 845-855

[13] Zhao J F, Li Z D, Li H X and Li J 2010 Thermocapillary migration of deformable bubbles at moderate to large Marangoni number in microgravity. Microgravity Sci. Tech. 22(3): 295-303

[14] Ma C and Bothe D 2011 Direct numerical simulation of thermocapillary flow based on the volume of fluid method. Int. J. Multiphase Flow 37(9): 1045-1058

[15] Borcia R and Bestehorn M 2007 Phase-field simulations for drops and bubbles. Phys. Rev. E 75(5): 056309

[16] Chakraborty I, Biswas G and Ghoshdastidar P 2013 A coupled level-set and volume-of-fluid method for the buoyant rise of gas bubbles in liquids. Int. J. Heat Mass Transfer 58(1): 240-259

[17] Liu H, Valocchi A, Zhang Y and Kang Q 2013 Phase-fieldbased lattice Boltzmann finite-difference model for simulating thermocapillary flows. Phys. Rev. E 87(1): 013010

[18] Zhang L, Yang C and Mao L S 2010 Numerical simulation of a bubble rising in a shear-thinning fluids. J. Non-Newtonian Fluid Mech. 165: 555-567

[19] Terasaka K and Tsuge H 2001 Bubble formation at a nozzle submerged in viscous liquids having yield stress. Chem. Eng. Sci. 56: 3237-3245

[20] Bhaga D and Weber 1981 M E Bubbles in viscous liquids: shapes, wakes and velocities. J. Fluid Mech. 105: 61-85

[21] Premlata A R, Tripathi M K and Sahu K C 2015 Dynamics of rising bubble inside a viscosity-stratified medium. Phys. Fluids 27: 072105.

[22] Tsamopoulos J, Dimakopoulos Y, Chatzidai N, Karapetsas G and Pavlidis M 2008 Steady bubble rise and deformation in Newtonian and viscoplastic fluids and conditions for bubble entrapment. J. Fluid Mech. 601: 123-164

[23] Taylor T and Acrivos A 1964 On the deformation and drag of a falling viscous drop at low Reynolds number. J. Fluid Mech. 18: 466-476

[24] Antal S, Lahey Jr R and Flaherty J 1991 Analysis of phase distribution in fully developed laminar bubbly two-phase flow. Int. J. Multiphase Flow 17: 635-652

[25] Sussman M and Puckett E G 2000 A coupled level set and volume-of-fluid method for computing 3D and axisymmetric incompressible two-phase flows. J. Comput. Phys 162: 301-337

[26] Hua J, Stene J F and Lin P 2008 Numerical simulation of 3D bubbles rising in viscous liquids using a front tracking method. J. Comput. Phys 227: 3358-3382

[27] Baltussen M, Kuipers J and Deen N 2014 A critical comparison of surface tension models for the volume of fluid method. Chem. Eng. Sci. 109: 65-74

[28] Han J and Tryggvason G 1999 Secondary breakup of axisymmetric liquid drops. I. acceleration by a constant body force. Phys. Fluids 11: 3650-3667

[29] Tripathi M K, Sahu K C and Govindarajan R 2014 Why a falling drop does not in general behave like a rising bubble. Sci. Rep. 4: 4771

[30] Hadamard J 1911 Mouvement permanent lent dune sphere liquide et visqueuse dans un liquide visqueux. CR Acad. Sci 152: $1735-1738$

[31] De Vries A, Biesheuvel A and Van Wijngaarden L 2002 Notes on the path and wake of a gas bubble rising in pure water. Int. J. Multiphase Flow 28: 18231835
[32] Shew W L and Pinton J 2006 Dynamical model of bubble path instability. Phys. Rev. Lett. 97: 144508

[33] Manga M and Stone H 1995 A Low Reynolds number motion of bubbles, drops and rigid spheres through fluidfluid interfaces. J. Fluid Mech. 287: 279-298

[34] Bonhomme R, Magnaudet J, Duval F and Piar B 2012 Inertial dynamics of air bubbles crossing a horizontal fluidfluid interface. J. Fluid Mech. 707: 405-443

[35] Kemiha M, Olmos E, Fei W, Poncin S and Li H Z 1991 Passage of a gas bubble through a liquid-liquid interface. Ind. Eng. Chem. Res. 46: 6099-6104

[36] Subramanian R S 1981 Slow migration of a gas bubble in a thermal gradient. AIChE J. 27(4): 646654

[37] Subramanian R S 1983 Thermocapillary migration of bubbles and droplets. Adv. Space Res. 3(5): 145-153

[38] Balasubramaniam R and Subramaniam R S 1996 Thermocapillary bubble migrationthermal boundary layers for large Marangoni numbers. Int. J. Multiphase Flow 22(3): 593-612

[39] Balasubramaniam R and Subramanian R S 2000 The migration of a drop in a uniform temperature gradient at large Marangoni numbers. Phys. Fluids 12(4): 733-743

[40] Crespo A, Migoya E and Manuel F 1998 Thermocapillary migration of bubbles at large Reynolds numbers. Int. J. Multiphase Flow 24(4): 685-692

[41] Merritt R M, Morton D S and Subramanian R S 1993 Flow structures in bubble migration under the combined action of buoyancy and thermocapillarity. J. Colloid Interf. Sci. 155(1): 200-209

[42] Zhang L, Subramanian R S and Balasubramaniam R 2001 Motion of a drop in a vertical temperature gradient at small marangoni-number the critical role of inertia. J. Fluid Mech. 448: 197-211

[43] Vochten R and Petre G 1973 Study of heat of reversible adsorption at air-solution interface II experimental determination of heat of reversible adsorption of some alcohols. $J$. Colloid Interface Sci. 42: 320-327

[44] Petre G and Azouni M A 1984 Experimental evidence for the minimum of surface tension with temperature at aqueous alcohol solution air interfaces. J. Colloid Interface Sci. 98: 261-263

[45] Petre G and Legros J C 1986 Thermocapillary movements under at a minimum of surface tension. Naturwissenschaften 73: $360-362$

[46] Savino R, Cecere A and Paola R D 2009 Surface tension driven flow in wickless heat pipes with self-rewetting fluids. Int. J. Heat Fluid Flow 30: 380-388

[47] Savino R, Cecere A, Vaerenbergh S V, Abe Y, Pizzirusso G, Tzevelecos W, Mojahed M and Galand Q 2013 Some experimental progresses in the study of the self-rewetting fluids for the selene experiment to be carried in the thermal platform 1 hardware. Acta Astronautica 89: 179-188

[48] Abe Y, Iwasaki A and Tanaka K 2004 Microgravity experiments on phase change of self-rewetting fluids. Ann. N.Y. Acad. Sci. 1027: 269285

[49] Sahu K C and Matar O K 2010 Three-dimensional linear instability in pressure-driven two-layer channel flow of a Newtonian and a Herschel-Bulkley fluid. Phys. Fluids 22: 112103

[50] Bird R B, Dai G C and Yarusso B J 1982 The rheology and flow of viscoplastic materials. Rev. Chem. Eng. 1: 1-70

[51] Barnes H A 1999 The yield stress - a review - everything flows? J. Non-Newtonian Fluid Mech. 81: 133-178 
[52] Bingham E C 1922 Fluidity and plasticity. New York: McGraw-Hill

[53] Herschel W H and Bulkley P 1926 Konsistenzmessungen von gummi-benzollsungen. Kolloid Z. 39: 291-300

[54] Frigaard I and Nouar C 2005 On the usage of viscosity regularisation methods for visco-plastic fluid flow computation. J. Non-Newtonian Fluid Mech. 127: 1-26

[55] Swain P A P, Karapetsas G, Matar O K and Sahu K C 2015 Numerical simulation of pressure-driven displacement of a viscoplastic material by a Newtonian fluid using the lattice Boltzmann method. Eur. J. Mech. B-Fluid 49: 197-207

[56] Tripathi M K, Sahu K C, Karapetsas G and Matar O K 2015 Bubble rise dynamics in a viscoplastic material. J. NonNewtonian Fluid Mech. 222: 217-226

[57] Astarita G and Apuzzo G 1965 Motion of gas bubbles in nonNewtonian liquids. AIChE J. 11(5): 815820

[58] Dubash N and Frigaard I 2007 Propagation and stopping of air bubbles in carbopol solutions. J. Non-Newtonian Fluid Mech. 142: 123-134

[59] Bhavaraju S M, Mashelkar R A and Blanch H W 1978 Bubble motion and mass transfer in non-Newtonian fluids: Part I. single bubble in power law and bingham fluids. AIChE J. 24: 1063-1070

[60] Papanastasiou T C 1987 Flows of materials with yield. $J$. Rheol. 31: 385-404

[61] Dimakopoulos Y, Pavlidis M and Tsamopoulos J 2013 Steady bubble rise in Herschel-Bulkley fluids and comparison of predictions via the augmented Lagrangian method with those via the Papanastasiou model. J. Non-Newtonian Fluid Mech. 200: 34-51

[62] Potapov A, Spivak R,Lavrenteva O M and Nir A 2006 Motion and deformation of drops in Bingham fluid. Ind. Eng. Chem. Res. 45(21): 6985-6995
[63] Singh J P and Denn M M 2008 Interacting two-dimensional bubbles and droplets in a yield-stress fluid. Phy. Fluids 20: 040901

[64] Kulkarni A A and Joshi J B 2005 Bubble formation and bubble rise velocity in gas-liquid system: a review. Ind. Eng. Chem. Res. 44: 5874-5931

[65] Kishore N, Chhabra R P and Eswaran V 2007 Drag on a single fluid sphere translating in power-law liquids at moderate Reynolds numbers. Chem. Eng. Sci. 62: 2422-2434

[66] Chhabra R S 1993 Bubbles drops and particles in nonNewtonian fluids. Boca Raton. CRC Press

[67] Chhabra R P 2006 Bubbles, drops, and particles in nonNewtonian fluids. CRC Press

[68] Ohta M, Iwasaki E, Obata E and Yoshida Y 2003 A numerical study of the motion of a spherical drop rising in shear-thinning fluid system. J. Non-Newtonian Fluid Mech. 113: 95-111

[69] Ohta M, Iwasaki E, Obata E and Yoshida Y 2005 Dynamics processes in a deformed drop rising through shear-thinning fluids. J. Non-Newtonian Fluid Mech. 132: 100-117

[70] Pillapakkam S B and Singh P 2001 A level-set method for computing solutions to viscoelastic two-phase flow. $J$. Comput. Phys 174: 552-578

[71] Wagner A J, Giraud L and Scott C E 2000 Simulation of a cusped bubble rising in a viscoelastic fluid with a new numerical method. Comput. Phys. Commun. 129: 227-232

[72] Vlez-Cordero J R, Smano D, Yue P, Feng J J and Zenit 2011 $\mathrm{R}$ Hydrodynamic interaction between a pair of bubbles ascending in shear-thinning inelastic fluids. J. Non-Newtonian Fluid Mech. 166(1): 118-132

[73] Islam M T, Ganesan P and Cheng Ji 2015 A pair of bubbles rising dynamics in a xanthan gum solution: a CFD study. RSC Adv. 5: 7819-7831. 